\title{
Terahertz wave emission from intrinsic Josephson junctions in high- $T_{c}$ superconductors
}

\author{
L Ozyuzer ${ }^{1,2,6}$, Y Simsek ${ }^{2}$, H Koseoglu ${ }^{1}$, F Turkoglu ${ }^{1}$, C Kurter ${ }^{3}$, \\ U Welp ${ }^{3}$, A E Koshelev ${ }^{3}$, K E Gray ${ }^{3}$, W K Kwok ${ }^{3}$, T Yamamoto ${ }^{4}$, \\ K Kadowaki $^{4}$, Y Koval ${ }^{2}$, H B Wang ${ }^{5}$ and P Müller ${ }^{2}$ \\ ${ }^{1}$ Department of Physics, Izmir Institute Technology, 35430 Izmir, Turkey \\ ${ }^{2}$ Department of Physics, University of Erlangen-Nurnberg, Germany \\ ${ }^{3}$ Materials Science Division, Argonne National Laboratory, IL, USA \\ ${ }^{4}$ University of Tsukuba, Tsukuba, Japan \\ ${ }^{5}$ National Institute for Materials Science, Tsukuba, Japan \\ E-mail: ozyuzer@iyte.edu.tr
}

Received 15 June 2009, in final form 4 September 2009

Published 20 October 2009

Online at stacks.iop.org/SUST/22/114009

\begin{abstract}
Recently, we experimentally demonstrated that rectangular mesa structures of intrinsic Josephson junctions (IJJ) in $\mathrm{Bi}_{2} \mathrm{Sr}_{2} \mathrm{CaCu}_{2} \mathrm{O}_{8+d}$ ( $\mathrm{Bi2212}$ ) can be used as a compact solid-state generator of continuous, coherent and polarized terahertz $(\mathrm{THz})$ radiation. In the present work, we will exhibit tall mesas (over 600 junctions) which were fabricated using UV lithography, e-beam lithography with photoresist and e-beam lithography with a Ti selective etching technique. We will present measurements of the $c$-axis resistance as a function of temperature and of current-voltage characteristics of $\mathrm{THz}$ emitting mesas with lateral sizes ranging from $30 \times 300$ to $100 \times 300 \mu \mathrm{m}^{2}$. Furthermore, we will discuss the dependence of the characteristics of the mesa structures on the oxygen doping level of the Bi2212 crystals. We will also experimentally show that the voltage-frequency relation of the ac Josephson effect has to match the cavity resonance for successful emission.
\end{abstract}

(Some figures in this article are in colour only in the electronic version)

\section{Introduction}

Terahertz sensing and imaging is a rapidly developing technology with wide-ranging applications including security, medicine, quality control and environmental monitoring [1]. There is a need for compact, continuous wave (CW), solidstate terahertz wave sources with practical emission powers. There are some available $\mathrm{CW}$ sources such as quantum cascade lasers that only work above $1.2 \mathrm{THz}$ [2]. Multiplication of Gunn diodes produces low output powers. Backward wave oscillators are bulky and have low output power at higher frequencies. Optically pumped $\mathrm{THz}$ gas lasers are also bulky and not tunable.

The ac Josephson effect allows for the construction of voltage to frequency converters that have great potential for

6 Address for correspondence: Department of Physics, Izmir Institute of Technology, Gulbahce Campus, Urla, 35430 Izmir, Turkey. the THz frequency range. The ac Josephson effect arises when a voltage bias is established across a Josephson junctiontwo superconducting electrodes separated by a thin insulating layer. An ac current appears that oscillates at the Josephson frequency $f_{\mathrm{J}}=V / \Phi_{o} . \quad V$ is the voltage across the junction and $\Phi_{o}$ is the flux quantum. According to this equation, $1 \mathrm{mV}$ corresponds to an emission frequency of $0.483 \mathrm{THz}$ for a single junction. Unfortunately, the observed emission power is very small for a single junction, i.e. of the order of $\mathrm{pW}[3,4]$. Enhanced emission power can be obtained from junction arrays if the oscillations of all the junctions in the array can be synchronized. Furthermore, low $T_{\mathrm{c}}$ superconductors have small energy gaps that limit the operational frequency of the Josephson oscillator. On the other hand, high temperature superconductors (HTSs) have large energy gaps that can support $\mathrm{THz}$ emission as described below. 
Certain HTS materials, e.g. $\mathrm{Bi}_{2} \mathrm{Sr}_{2} \mathrm{CaCu}_{2} \mathrm{O}_{8+\delta}$ (Bi2212), form a highly anisotropic layered structure in which the superconducting $\mathrm{CuO}_{2}$ bi-layers are separated by intervening insulating $\mathrm{Bi}-\mathrm{Sr}-\mathrm{O}$ layers, resulting in a stack of so-called intrinsic Josephson junctions (IJJs) [5]. Since the IJJs are naturally stacked on the atomic scale along the $c$ axis of Bi2212 single crystals, highly uniform junction arrays of unsurpassed density arise which hold the potential for very high emission power [6]. There have been many attempts to obtain $\mathrm{THz}$ radiation from layered superconductors using IJJ properties. Iguchi et al observed microwave emission from quasiparticleinjected nonequilibrium high temperature superconductors [7]. Bae et al detected the emission of radiation from flux flow motion in Bi2212 under a strong magnetic field [8]. Batov et al observed emission up to $0.5 \mathrm{THz}$ from Bi2212 with a weak power [9] probably due to unsynchronized Josephson junctions.

For the observation of $\mathrm{THz}$ wave emission under zero magnetic fields [10], the Bi2212 mesas were designed as electromagnetic resonators-cavities-and strong $\mathrm{THz}$ emission was observed when the voltage across the mesa was adjusted in such a way that the Josephson frequency of the IJJ matches the cavity resonance. The fundamental frequencies of the observed emission were as high as $0.85 \mathrm{THz}$ and radiation power was up to $0.5 \mu \mathrm{W}$, which has not been achieved before. We showed that the emission frequency is proportional to $1 / w$, where $w$ is the width of the mesa indicating that the cavity resonance synchronizes the oscillations of the IJJ, resulting in strong radiation. These studies stimulated further work on $\mathrm{THz}$ generation from intrinsic Josephson junctions in Bi2212 mesas and recently one order of magnitude larger radiation power $(\sim 5 \mu \mathrm{W})$ and higher harmonics (up to fourth order, corresponding to $2.5 \mathrm{THz}$ ) have been obtained [11]. Tunability of the $\mathrm{THz}$ emission and local temperature properties have also been studied [12-14].

Many theoretical models have been proposed to explain the IJJ effect $[15,16]$ and the mechanism of $\mathrm{THz}$ wave generation from Bi2212 mesas [17-26]. One model is based on the modulation of the Josephson critical current density along the width of the mesa, which allows for the efficient excitation of the cavity resonance under zero magnetic fields [17]. In another model [19, 20], a new dynamic state has been discovered in which a phase kink occurs, enabling the excitation of the resonance. A recent suggestion is that the nonradiative component of the oscillating magnetic field of the cavity resonance mode determines the $\mathrm{THz}$ emission [24].

Currently, there is no consensus and the need for more complete experimental characterization of the properties of $\mathrm{THz}$ emitting Bi2212 mesas exists. In this paper, we will present common properties such as resistance versus temperature $(R-T)$ and $I-V$ of radiating underdoped mesas, as well as of overdoped and optimally doped ones. In one of the radiating mesas, it will be shown that $\mathrm{THz}$ wave emission occurs only when the cavity resonance and applied voltage satisfies the Josephson voltage-frequency relation.

\section{Experiment}

Bi2212 single crystals have been grown by the floating zone method. In order to obtain various doping levels, we annealed the crystals at $600{ }^{\circ} \mathrm{C}$ in vacuum or under Ar flow at $400{ }^{\circ} \mathrm{C}$ or in air. Crystals of a typical size of $1 \times 1 \mathrm{~mm}^{2}$ were glued on sapphire substrates with silver epoxy. The crystals were cleaved using scotch tape and immediately coated with a $100 \mathrm{~nm}$ thick Au film evaporated under high vacuum. The rectangular mesa structures $\left(30 \times 300\right.$ to $\left.100 \times 300 \mu \mathrm{m}^{2}\right)$ were fabricated using Ar-ion beam etching. We employed three different methods to form etch masks for the pattern transfer into the Bi2212 crystal. One of them is based on the fabrication of photoresist patterns on the Bi2212 crystal using UV optical lithography with glass shadow masks. In the second technique we used electron beam lithography to pattern the AZ-5214 (Clariant) photoresist. In the third masking technique, we used $300 \mathrm{~nm}$ thick Ti layers as hard etch masks for the Ar-ion milling step. Ti patterns were created by lift-off and electron beam lithography. The ratio of the etching rate of $\mathrm{Ti} / \mathrm{Bi} 2212$ is 0.3 for Ar-ion beam with collimated optics under $250 \mathrm{eV}$ beam energy and $10 \mathrm{~mA}$ beam current. The production of mesas using reactive ion etching is under investigation for achieving higher etching selectivity. Since the photoresist does not stand well being etched with the ion beam over long etching periods, e.g. $60 \mathrm{~min}$ for $1 \mu \mathrm{m}$, the samples were cooled by liquid $\mathrm{N}_{2}$ during the etching process. Furthermore, the cooling prevents the Bi2212 from over-heating that might cause a doping level change [27]. After etching, we have obtained large and tall mesas whose planar dimensions were between $30 \times 300$ and $100 \times 300 \mu \mathrm{m}^{2}$, and their heights were approximately $1 \mu \mathrm{m}$. In order to make a contact on top of the mesas, firstly an insulating layer $\left(\mathrm{CaF}_{2}\right)$ was evaporated on part of the mesa and the Bi2212 single crystal. After that, a gold stripe was created by a shadow mask or lift-off technique on the mesa and $\mathrm{CaF}_{2}$ layers (figure 1).

In order to characterize the Bi2212 mesas, $c$-axis resistance versus temperature $(R-T)$, and current-voltage behavior $(I-V)$ were measured in an He flow cryostat equipped with an optical window as shown in figure 2. During $I-V$ characterization, we used an Si composite bolometer to detect the emission power (figure 2). As the applied current through the IJJ stacks was slowly swept by a function generator, the emission power from the long side of the mesa has been simultaneously monitored by the $\mathrm{Si}$ composite bolometer. Since $\mathrm{THz}$ radiation is strongly absorbed by water vapor, we used a dry $\mathrm{N}_{2}$ box between the cryostat and the bolometer.

\section{Results}

We have already shown that the $\mathrm{THz}$ emission is due to a geometric assurance that the sides of the mesas are important because the impedance mismatch between Bi2212 and vacuum might affect the electromagnetic wave released from the mesa. We usually employ atomic force microscopy (AFM) imaging for mesa height and characterization of the slope of the long side faces. Figure 3 shows an AFM image of one of the $\mathrm{THz}$ emitting mesas fabricated using photoresist patterned with a 


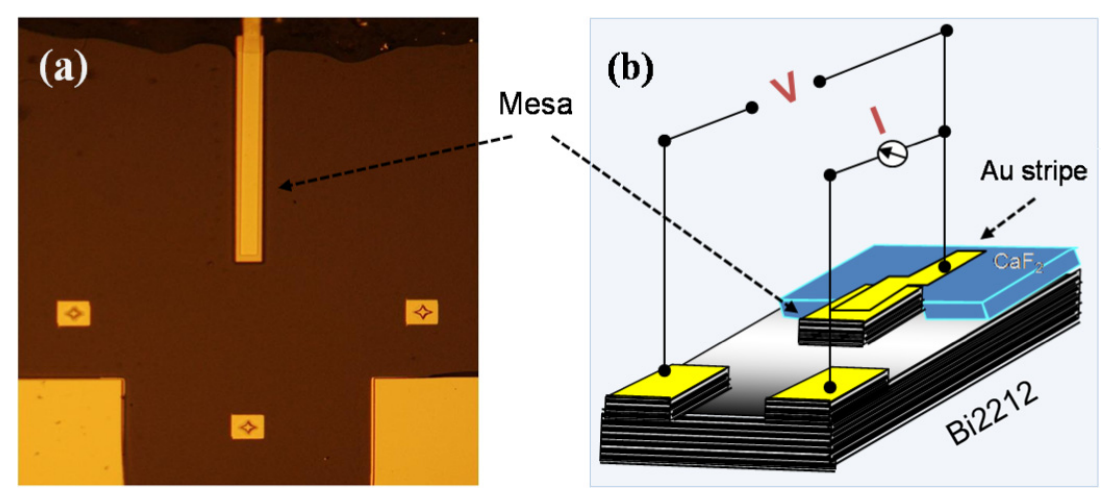

Figure 1. Mesa structure on Bi2212. (a) Optical image of $30 \times 300 \mu \mathrm{m}^{2}$ mesa with contact pads. (b) Measurement configuration of mesa structure with three-point probe.

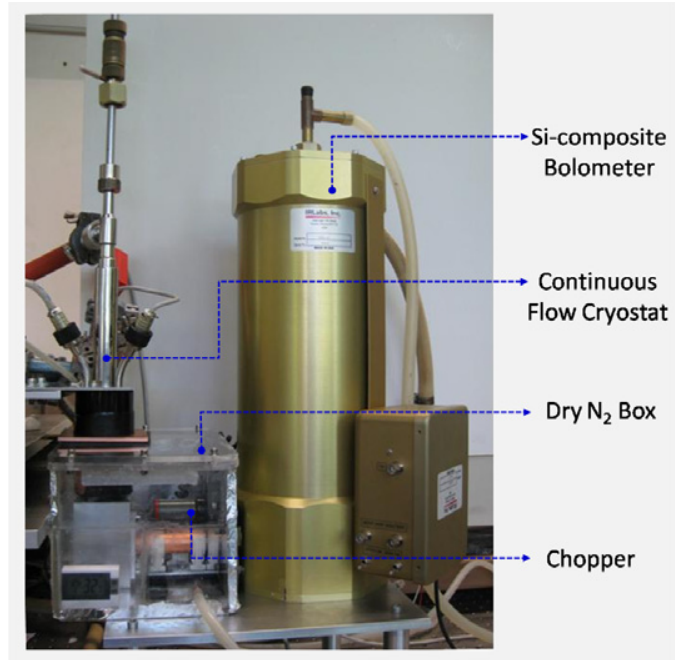

Figure 2. Experimental setup including sample-in-vacuum continuous flow cryostat, chopper and Si composite bolometer.

shadow mask and UV lithography. Figure 3 indicates that there is an angle as high as $45^{\circ}$ between the basal plane and the side of the mesa. After examining many mesas, e.g. photoresist angles before etching and mesa angles after etching, we found that the ion beam replicates the photoresist angles. While the trapezoidal (narrow top, wide bottom) mesa could be the origin of the frequency tunability of the $\mathrm{THz}$ emission [12], studies of the side surface roughness may reveal its influence on the synchronization of the oscillations in different layers and on the emission power. It is not clear yet how the actual cross section of the mesa affects the resonant condition and emission power of the mesa. We believe that, while right angles between the basal plane and the mesa sides should induce more intense power, the frequency tunability of the mesa may not be as large as for the trapezoidal cross section. Instead of a glass shadow mask, we also fabricated mesas using ebeam writing on regular photoresist and PMMA for lift-off technique. Figure 4(a) shows a mesa fabricated with e-beam writing and Ar beam etching. It displays a considerable surface roughness of the side faces of the mesa. The SEM image of a mesa fabricated by e-beam lithography and selective etching of Ti can be seen in figure 4(b). It is obvious that cleaner

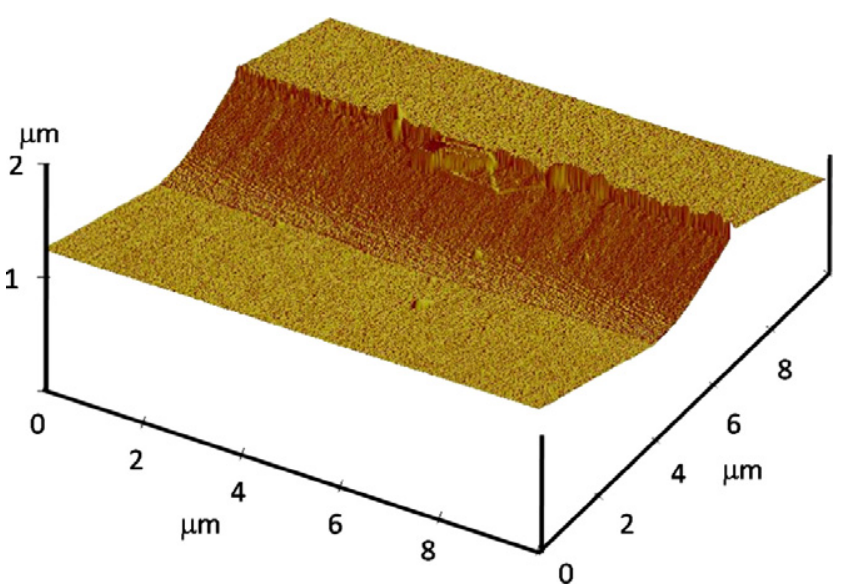

Figure 3. $10 \times 10 \mu \mathrm{m}^{2}$ AFM image of one of the radiating $100 \times 300 \mu \mathrm{m}^{2}$ mesas showing the edge profile.

mesas with sharp edges can be fabricated by selective etching. The THz emission characteristics of both mesa types are under investigation.

In order to understand the mechanism underlying the $\mathrm{THz}$ emission, we will emphasize generic features of radiating mesas. Figure 5 shows a comparison of $R-T$ measurements of THz emitting mesas with various widths. The onsets of critical temperatures of the mesas are between 75 and $87 \mathrm{~K}$, consistent with the underdoped state of the underlying Bi2212 crystals. All $R-T$ characteristics exhibit semiconductor-like behavior above $T_{\mathrm{c}}$, as expected for underdoped Bi2212 [28]. Due to our three-point measurement configuration, the $c$-axis resistivity cannot be exactly determined. On the other hand, assuming that the contact resistance is weakly dependent on temperature above $T_{\mathrm{c}}$, the $R\left(T_{\mathrm{c}}\right) / R(300 \mathrm{~K})$ values from figure 5 give at least a hint. In figure 5, the contact resistance can be seen in our data below $T_{\mathrm{c}}$ due to our three-point measurements: it is usually $<5 \Omega$ except for one sample $(w=40 \mu \mathrm{m})$. A recent study on $10 \times 10 \mu \mathrm{m}^{2}$ mesas show the contact resistance to be a superconductor-insulator-normal-metal junction that can be expected to be weakly temperature-dependent above $T_{\mathrm{c}}$ and diverge at low temperatures [29]. Values range from 0.00015 to $0.0005 \Omega \mathrm{cm}^{2}$. This gives a resistance of $1-$ $4 \Omega$ for a $40 \times 300 \mu \mathrm{m}^{2}$ mesa, consistent with our data. 


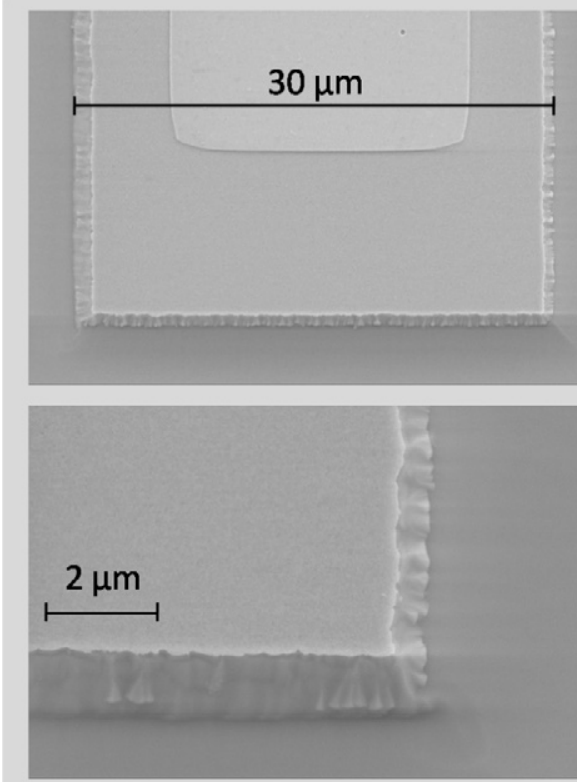

(a)

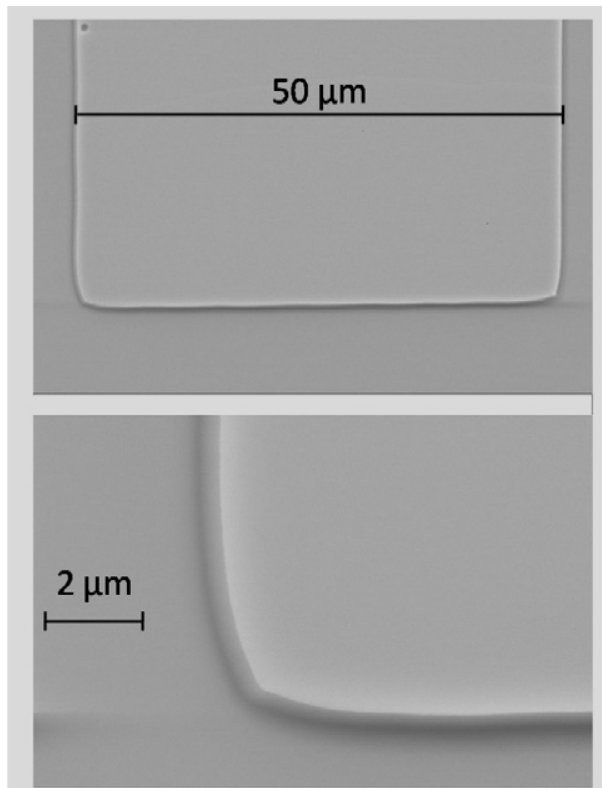

(b)

Figure 4. SEM images of mesas fabricated using e-beam lithography and different masking techniques. (a) Photoresist mask and (b) Ti mask. The heights of mesas in (a) and (b) are 1.2 and $0.7 \mu \mathrm{m}$, respectively.

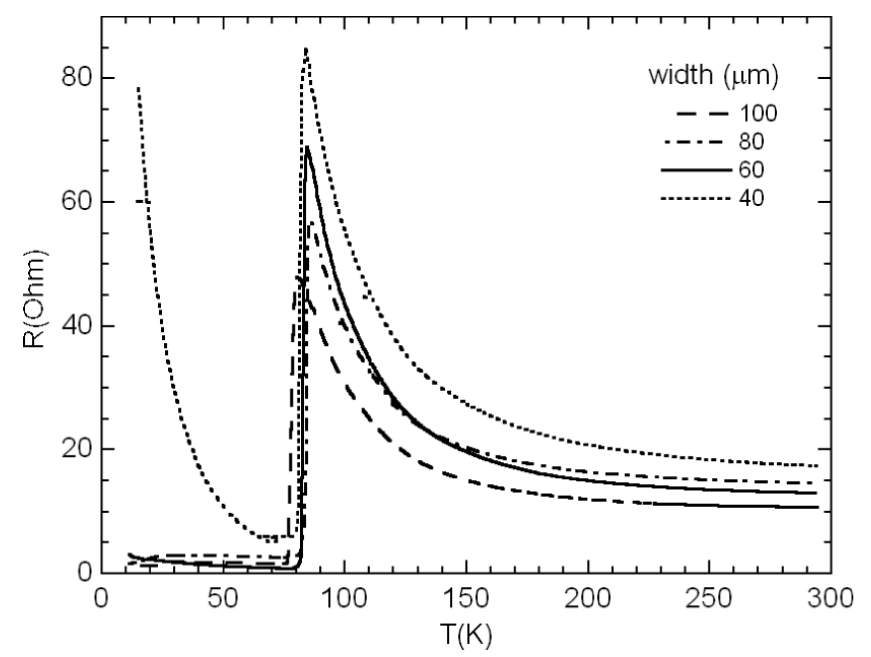

Figure 5. Resistance versus temperature behavior of $\mathrm{THz}$ emitting mesas with various widths. A constant current of $I=10 \mu \mathrm{A}$ was used. Note that the resistance below $T_{\mathrm{c}}$ is due to contact resistance.

As seen in figure 5, all $R-T$ characteristics exhibit $R\left(T_{\mathrm{c}}\right) / R$ $(300 \mathrm{~K})>4$ for $\mathrm{THz}$ emitting mesas. Since the oxygen doping level dependence of the $c$-axis resistivity of Bi2212 was systematically studied by Watanabe et al [28], $R\left(T_{\mathrm{c}}\right) / R$ $(300 \mathrm{~K})>4$ indicates a necessity of a certain doping range of $\delta \sim 0.22$ for $\mathrm{THz}$ emission.

Figure 6 shows $I-V$ curves of THz emitting mesas on the same plot. All measurements have been taken below $12 \mathrm{~K}$. The current is swept back and forward many times to obtain each $I-V$ curve. Some of the quasiparticle branches can be seen. All of our radiating samples have a Josephson critical current density of about $100 \mathrm{~A} \mathrm{~cm}^{-2}$. All of them show backbending

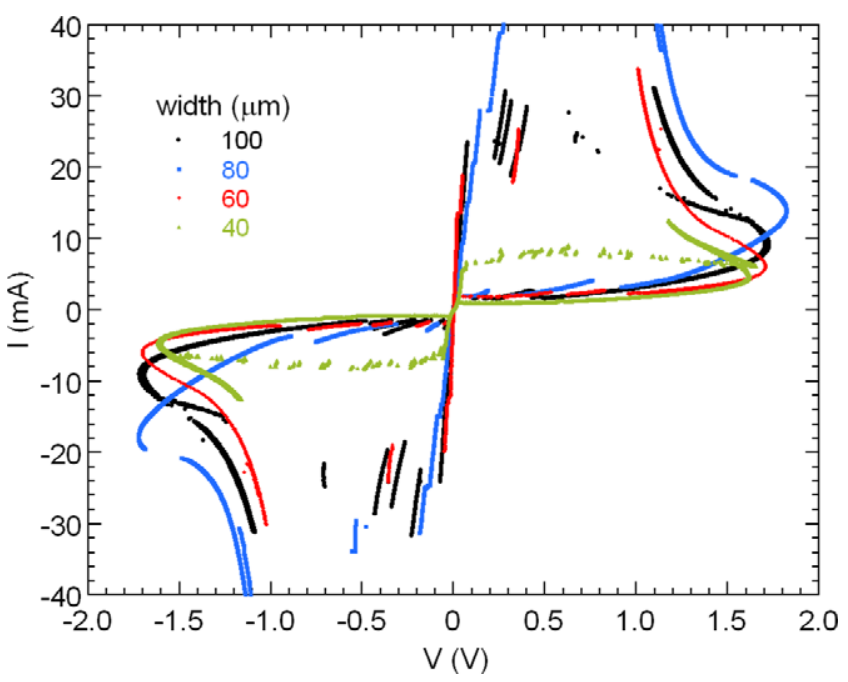

Figure 6. Current versus voltage behavior of $\mathrm{THz}$ emitting mesas with various widths. During the measurements the temperature was kept below $12 \mathrm{~K}$ for all curves.

above $1.5 \mathrm{~V}$ where heating severely affects the local mesa temperature [30, 31]. A voltage of $1.5 \mathrm{~V}$ corresponds to $2 \mathrm{mV}$ per junction, which indicates that a fundamental frequency of $1 \mathrm{THz}$ may be possible before heating severely affects the mesa. The backbending voltage of large area mesas depends on various parameters such as doping level of Bi2212, number of junctions and surface area of the mesa [13]. It has been shown that in IJJ stacks the heating can be precluded by lowering the number of junctions and by decreasing the mesa area. Unfortunately, these also decrease the power and conflict with the resonance condition in our case. However, 


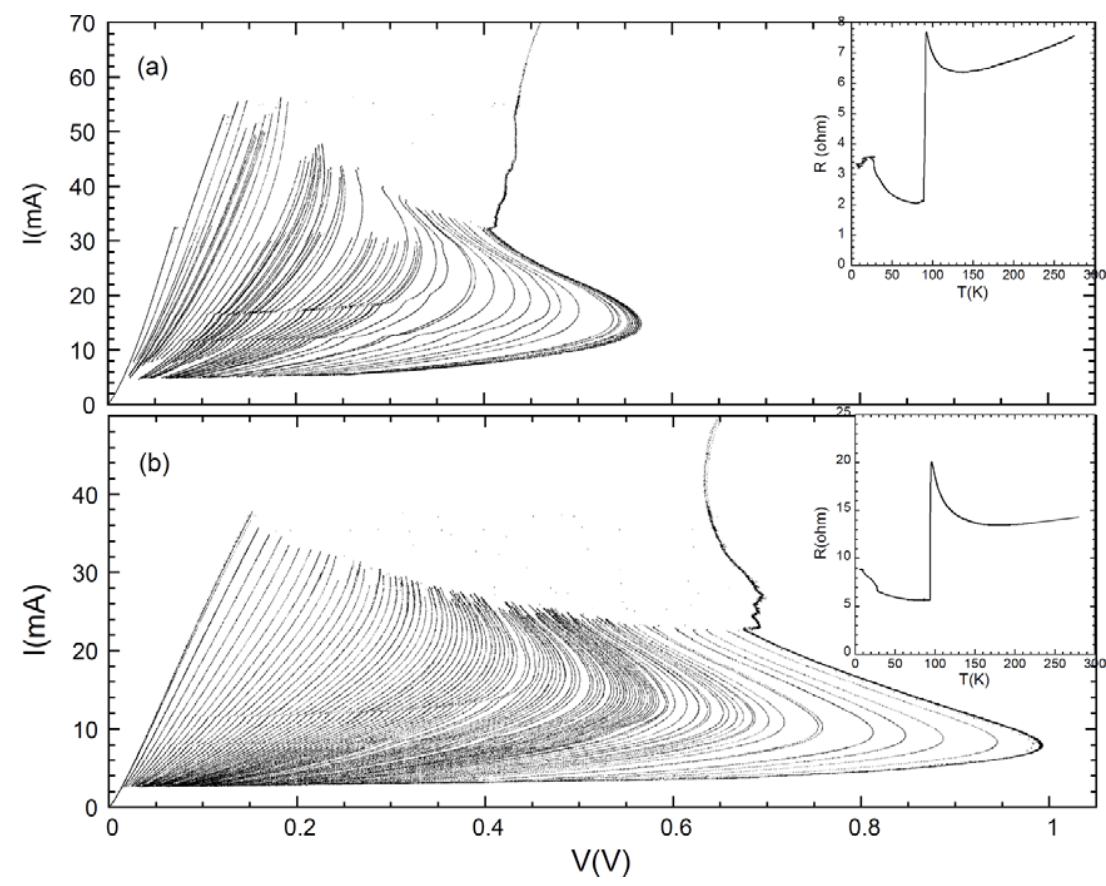

Figure 7. $I-V$ characteristics of overdoped (a) and optimally doped (b) mesas at $4.2 \mathrm{~K}$. Some of the quasiparticle branches are recorded using manual scan of the current source.

recent calculations suggest that circular mesas excite a higher frequency than rectangular ones [21] for the same lateral size.

We also investigated large area mesas fabricated from overdoped and optimally doped Bi2212 crystals. Figures 7(a) and (b) show $I-V$ and $R-T$ measurements of these samples. Both mesas have $50 \times 300 \mu \mathrm{m}^{2}$ area and $1.0 \mu \mathrm{m}$ height. The comparison of $R-T$ behaviors (insets of figure 7) indicates different oxygen concentrations of the samples. Here $R\left(T_{\mathrm{c}}\right) /$ $R(300 \mathrm{~K})$ values are 1.0 and 1.3 for slightly overdoped and optimally doped samples, respectively. Furthermore, the $I-$ $V$ curves indicate that the Josephson critical current densities are 2-3 times larger than the $\mathrm{THz}$ emitting mesas presented in figure 6. While both samples have the same number of layers, overdoped samples show backbending around $0.6 \mathrm{~V}$. In figure 7(b), the optimally doped sample shows backbending at $1.0 \mathrm{~V}$, that is $30 \%$ smaller than underdoped mesas given in figure 6. This behavior can be expected since the $c$-axis quasiparticle conductivity - and therefore dissipation and self-heating at fixed voltage-increase significantly from underdoped to optimally doped to overdoped Bi2212. It has already been observed that the doping dependence of the energy gap in overdoped crystals is relatively smaller than in optimally doped and underdoped Bi2212 [32, 33]. This does not explain the lower voltage for backbending, because the separation between branches are already much smaller than the energy gap. The injection of quasiparticles in IJJs causes nonequilibrium and heating at high bias that has been studied extensively [34]. The width of $50 \mu \mathrm{m}$ corresponds to an emission frequency of roughly $0.7 \mathrm{THz}$ for these samples, which, according to the Josephson voltage-frequency relation, occurs at $0.87 \mathrm{~V}$ for 600 junctions. This value is inside the heating region as seen in figure 7 , where the heating causes local temperature increases seen in the $I-V$ curves of both mesas. We prepared many mesas out of these crystals and we did not detect $\mathrm{THz}$ emission. The optimally doped and overdoped mesas have too high a quasiparticle conductivity and too high a dissipation such that one cannot reach the voltage per junction that is required for $1 \mathrm{THz}$.

$\mathrm{THz}$ emission characteristics are obtained during $I-V$ scans and recorded simultaneously. This gives us information on how the $I-V$ looks like before and after $\mathrm{THz}$ emission. Figure 8 shows $\mathrm{THz}$ emission characteristics of one of the $100 \times 300 \mu \mathrm{m}^{2}$ mesas at $20 \mathrm{~K}$. This is one of the observed generic emission characteristics in rectangular mesas given in figure 6. At high bias $(> \pm 1.0 \mathrm{~V})$, the $I-V$ curve shows backbending and the bolometer detects the heating of the mesa in the form of unpolarized black body radiation [10], see figure $8(\mathrm{~b})$. When all the junctions are in the resistive state and the bias is slowly decreased, a THz emission peak can be seen (arrows in figure 8(b)). Finally a retrapping event occurs in which some IJJ switch back to the superconducting state. The retrapping is seen as a jump in the $I V$ curve accompanied by the sudden loss of emission. This loss of emission arises because the resonance condition is no longer fulfilled for the remaining resistive junctions. FTIR and cutoff filter measurements revealed an emission frequency of $0.35 \mathrm{THz}$ for $100 \mu \mathrm{m}$ wide mesas, which is in good agreement with the geometrical resonance and the ac Josephson relation [10].

For the first time, we will present additional evidence for our previous confirmation [10] that the Josephson voltagefrequency relation has to be satisfied for successful emission in rectangular mesas. Figure 9 is another $I-V$ and bolometer response $-V$ trace of the mesa given in figure 8 . The upper panel is the $I-V$ curve and the lower panel is the bolometer response versus voltage. When all junctions, roughly 600, are in the resistive state and the bias is decreasing slowly 


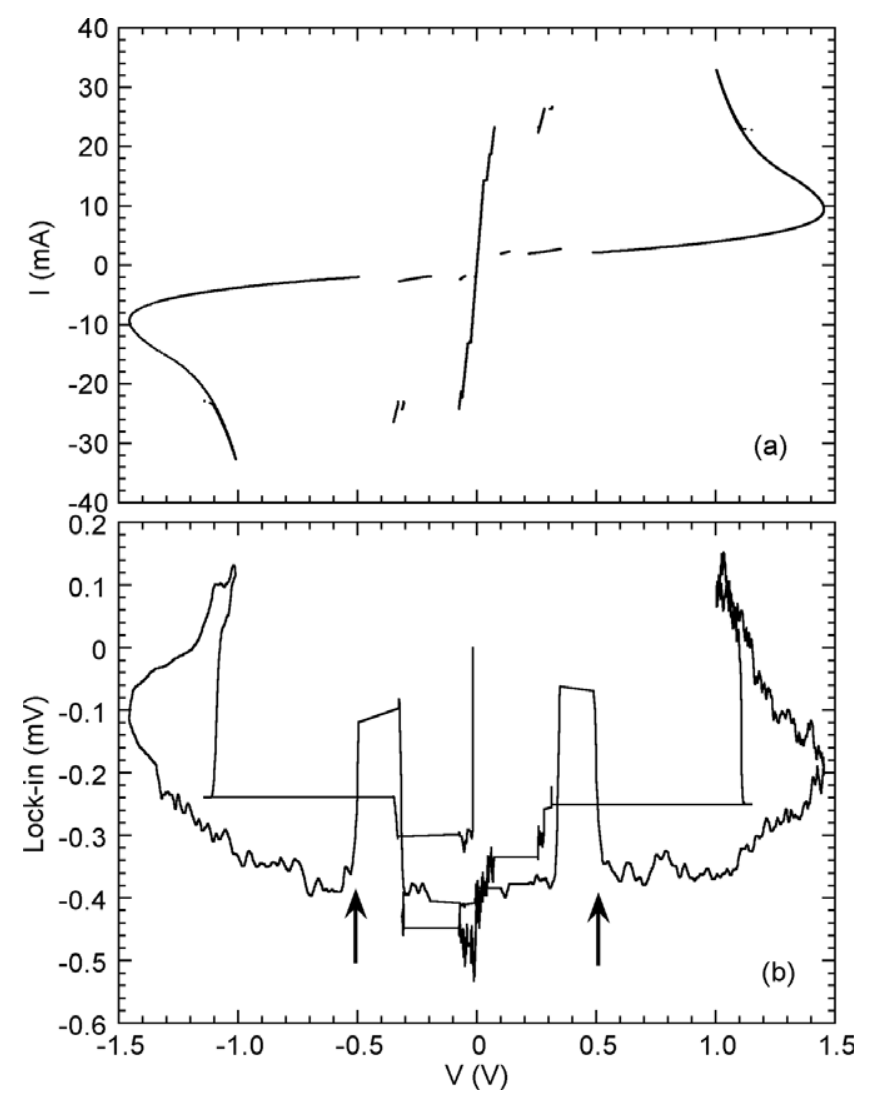

Figure 8. Current-voltage (a) and bolometer output versus voltage (b) graph of one of the $100 \times 300 \mu \mathrm{m}^{2}$ mesas at $20 \mathrm{~K}$. The arrows indicate the emission bias voltages.

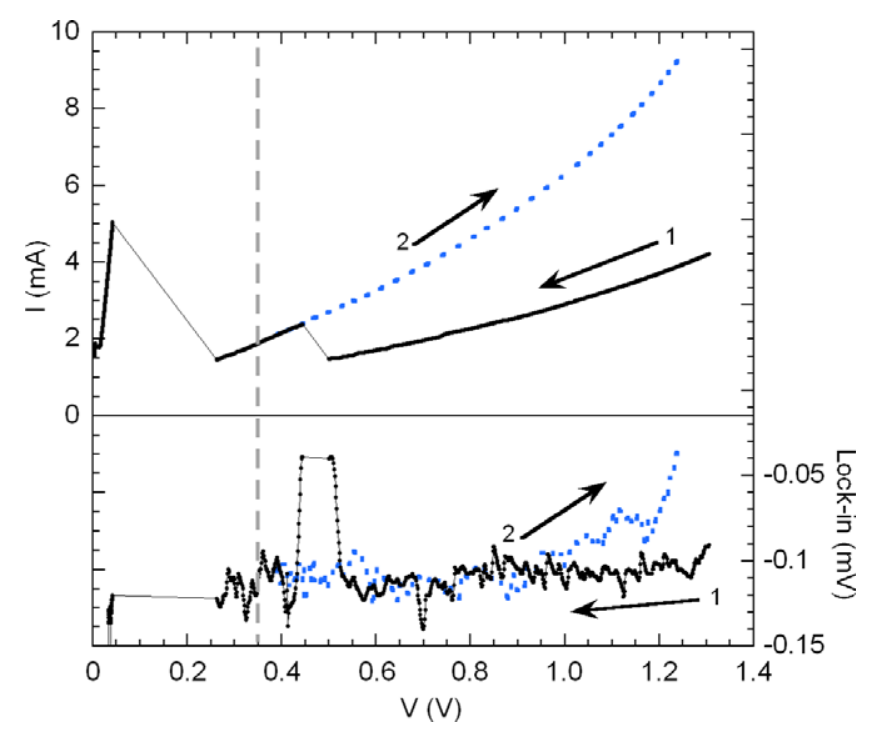

Figure 9. Another trace of current versus voltage and bolometer output versus voltage graph of the mesa given in figure 8 . At the position of the dashed vertical line, voltage is returned back after switching some of the resistive junctions to the superconducting state.

(direction of arrow 1), the bolometer begins to give a response at $0.53 \mathrm{~V}$. On further decreasing the bias, the signal reaches a peak position around $0.50 \mathrm{~V}$ and the $I-V$ curve shows a jump due to retrapping as described above. At the position of the light vertical dashed line, we reverse the direction of current sweep shown by arrow 2 . When we changed the bias directions to positive, such that the curve follows the dashed line, it passes the emission voltage, $0.50-0.53 \mathrm{~V}$, but the bolometer does not show any trace of emission. It is not possible to find any emission above $0.50 \mathrm{~V}$ for this trace, but it might be seen below it for this curve. After first retrapping, it is clear that the number of junctions in the resistive state is less than 600 . This confirms that the voltage per junction has to be appropriate for the resonance condition.

\section{Summary}

In this study, we presented data on tall (over 600 junctions) and large mesas $\left(30 \times 300\right.$ to $\left.100 \times 300 \mu \mathrm{m}^{2}\right)$ of Bi2212 fabricated by three different techniques; UV lithography, ebeam lithography with photoresist and e-beam lithography with Ti selective etching. It is shown that Ti selective etching creates more clear mesas with sharp edges. Currently, the effect of edge roughness on $\mathrm{THz}$ emission is not known and under investigation. We show that the $\mathrm{THz}$ emitting mesas are below a certain underdoped level, which has relatively small critical current in contrast to optimally doped and overdoped Bi2212. Therefore, large area mesas fabricated from underdoped crystals cause less heating and backbending takes place after the cavity resonance in voltage scale. Furthermore, we show that in IJJ mesas the voltage per junction has to be appropriate for a resonance condition.

\section{Acknowledgments}

This research is supported in part by the TUBITAK (Scientific and Technical Council of Turkey) project no. 108T238 and the US Department of Energy, Basic Energy Sciences-Materials Sciences, under contract no. DE-AC02-06CH11357. LO acknowledges support from the Turkish Academy of Sciences and the Alexander von Humboldt Foundation.

\section{References}

[1] Tonouchi M 2007 Nat. Photon. 197

[2] Wade A, Fedorov G, Smirnov D, Kumar S, Williams B S, $\mathrm{Hu} \mathrm{Q}$ and Reno J L 2009 Nat. Photon. 341

[3] Langenberg D N, Scalapino D J, Taylor B N and Eck R E 1965 Phys. Rev. Lett. 15294

[4] Yanson I K, Svistunov V M and Dmitrenko I M 1965 Zh. Eksp. Teor. Fiz. 48476

Yanson I K, Svistunov V M and Dmitrenko I M 1965 Sov. Phys.-JETP 21650 (Engl. Transl.)

[5] Kleiner R, Steinmeyer F, Kunkel G and Müller P 1992 Phys. Rev. Lett. 682394

[6] Koyama T and Tachiki M 1996 Solid State Commun. 96367

[7] Iguchi I 2000 Supercond. Sci. Technol. 1393

[8] Bae M H, Lee H J and Choi J H 2007 Phys. Rev. Lett. 98027002

[9] Batov I E, Jin X Y, Shitov S V, Koval Y, Müller P and Ustinov A V 2006 Appl. Phys. Lett. 88262504

[10] Ozyuzer L et al 2007 Science 3181291

[11] Kadowaki K et al 2008 Physica C 468634 
[12] Gray K E et al 2009 IEEE Trans. Appl. Supercond. 19886 (arXiv:0901.4290)

[13] Kurter C et al 2009 IEEE Trans. Appl. Supercond. 19428

[14] Wang H B, Guenon S, Yuan J, Iishi A, Arisawa S, Hatano T, Yamashita T, Koelle D and Kleiner R 2009 Phys. Rev. Lett. 102017006

[15] Sakai S, Bodin P and Pedersen N F 1993 J. Appl. Phys. 732411

[16] Kleiner R 1994 Phys. Rev. B 506919

[17] Koshelev A E and Bulaevskii L N 2008 Phys. Rev. B 77014530

[18] Lin S, Hu X and Tachiki M 2008 Phys. Rev. B 77014507

[19] Lin S and Hu X 2008 Phys. Rev. Lett. 100247006

[20] Koshelev A E 2008 Phys. Rev. B 78174509

[21] Hu X and Lin S 2008 Phys. Rev. B 78134510

[22] Matsumoto H, Koyama T, Machida M and Tachiki M 2008 Physica C 4681899

[23] Klemm R and Kadowaki K 2008 arXiv:0807.3082

[24] Tachiki M, Fukuya S and Koyama T 2009 Phys. Rev. Lett. 102127002
[25] Koyama T, Matsumoto H, Machida M and Kadowaki K 2009 Phys. Rev. B 79104522

[26] Rakhmanov A L, Savel'ev S E and Nori F 2009 Phys. Rev. B 79184504

[27] Nagai Y, Tsuru K, Terada A and Yanagisawa K 1991 IEEE Trans. Magn. 271622

[28] Watanabe T, Fuji T and Matsuda A 1997 Phy. Rev. Lett. 792113

[29] Kurter C, Zasadzinski J F, Ozyuzer L, Hinks D G and Gray K E 2009 Phys. Rev. B submitted

[30] Krasnov V M, Sandberg M and Zogaj I 2005 Phys. Rev. Lett. 94077003

[31] Wang H B, Hatano T, Yamashita T, Wu P H and Müller P 2005 Appl. Phys. Lett. 86023504

[32] Ozyuzer L, Zasadzinski J F and Miyakawa N 1999 Int. J. Mod. Phys. B 133721

[33] Ozyuzer L, Zasadzinski J F, Kendziora C and Gray K E 2000 Phys. Rev. B 613629

[34] Ozyuzer L, Kurter C, Zasadzinski J F, Gray K E, Hinks D G and Miyakawa N 2005 IEEE Trans. Appl. Supercond. 15181 and references therein 